\title{
Responsivity Enhanced NMOSFET Photodetector Fabricated by Standard CMOS Technology
}

\author{
Fuwei Wu, Xiaoli Ji, and Feng Yan \\ School of Electronic Science and Technology, Nanjing University, Nanjing 210046, China \\ Correspondence should be addressed to Xiaoli Ji; xji@nju.edu.cn and Feng Yan; fyan@nju.edu.cn
}

Received 30 October 2014; Revised 10 November 2014; Accepted 9 December 2014

Academic Editor: Jiwu Lu

Copyright ( 92015 Fuwei Wu et al. This is an open access article distributed under the Creative Commons Attribution License, which permits unrestricted use, distribution, and reproduction in any medium, provided the original work is properly cited.

\begin{abstract}
Increasing the responsivity is one of the important issues for a photodetector. In this paper, we demonstrate an improved NMOSFET photodetector by using deep-n-well (DNW) structure which can improve the responsivity of the photodetector significantly. The experimental results show that the responsivity can be enhanced greatly by the DNW structure and is much larger than the previous work when DNW is biased with $0.5 \mathrm{~V}$, while the dark current exhibits almost no increase. Further characterization indicates that the diode formed by the bulk and DNW can efficiently absorb photons and has a large gain factor of the photocurrent especially under low light condition, which gives a more promising application for the detector to detect the weak light.
\end{abstract}

\section{Introduction}

Image sensors based on standard CMOS technology have obtained great success in the market of mobile phones, digital cameras, and other consumer electronics, because of its low cost, low power consumption, and design flexibility [1-3]. However, in the application field with low illumination, such as biological florescence detection, weak light imaging, and astronomical observation, photodetectors with high sensitivity are necessary $[4,5]$. Previously, lateral BJT-based photodetectors have been reported, in which the source, drain, and bulk of a MOSFET form a bipolar transistor to amplify the photocurrent and enhance the responsivity [6]. Based on the same operation mode, Zhang reported a high gain gate-bulk tied NMOSFET photodetector on SOI substrate. In this photodetector, the drain/bulk junction diode absorbs photons and generates electron-hole pairs. The electrons are swept to the drain while the holes accumulate in the bulk which increases the bulk potential. Because of the gate-bulk tied structure, it is then fed back to the gate. The positive feedback leads to further turn-on of the MOSFET, supplying an amplified drain/bulk diode photocurrent to the outputs [7]. But because of the low photon-absorbing efficiency and the high cost of SOI substrate, this idea is extended to bulk structure of MOSFET by us. Here, a gate-bulk tied NMOSFET transistor on deep-n-well is fabricated by standard CMOS technology. Its photoelectric characteristics are investigated. The experimental results show that the DNW/bulk diode can absorb photons efficiently and improve the responsivity significantly when DNW is positively biased while the dark current keeps almost no change.

\section{Experiment}

Figure 1(a) shows the cross-section of the proposed NMOSFET photodetector. It is formed by a gate-bulk tied (GB tied) NMOSFET transistor on the deep-n-well. The detector is fabricated in standard CMOS process. The size $(W \times L)$ of NMOSFET is $10 \mu \mathrm{m} \times 0.55 \mu \mathrm{m}$ and the gate oxide thickness is $12 \mathrm{~nm}$ in this study. The depth of source and drain is about $0.2 \mathrm{um}$. And the depth of the DNW is about $0.8 \mathrm{um}$. The electrical characteristics of the photodetectors are measured using Keithley 4200 semiconductor characterization system in Cascade Summit 12000 probe station employing a $150 \mathrm{~W}$ Xe lamp as the illumination source to emit white light. During photodetecting, the gate and bulk are tied together and left floating, the source is grounded, and the drain and DNW are biased with a positive voltage. The output current is measured in the drain side.

As shown in Figure 1(a), because the NMOSFET is fabricated on a DNW, two photodiodes are formed by the 


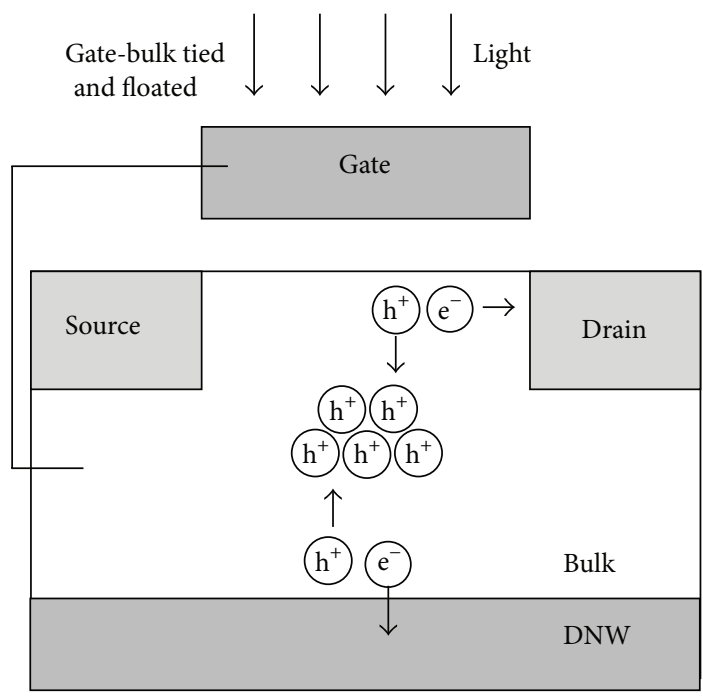

(a)

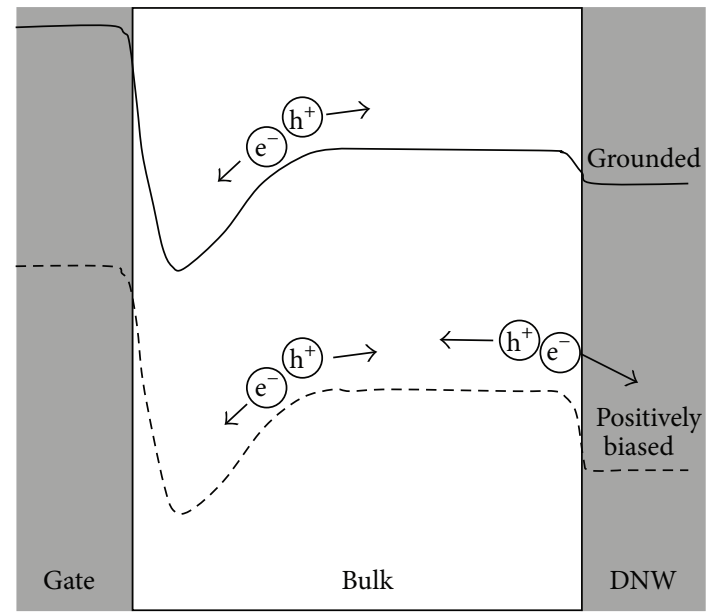

(b)

FIGURE 1: (a) The cross-section view of the responsivity enhanced NMOSFET photodetector under light condition. (b) The band diagram of the photodetector with different DNW voltages under light condition.

drain/bulk junction diode $\left(J_{1}\right)$ and the DNW/bulk junction diode $\left(J_{2}\right)$ during detecting. As discussed in [7], the $J_{1}$ can absorb photons during detecting and an amplified $J_{1}$ photocurrent is obtained in the drain side because of the GB tied structure. Meanwhile, $J_{2}$ can become the second source of the outputs when $J_{2}$ is reverse biased with a positive DNW voltage. Photons are absorbed in $J_{2}$ and the photogenerated holes are then injected into the bulk, which provides an additional increase of the gate potential as shown in Figure 1(b). As a result, the output photocurrent can be further increased.

Therefore, when DNW is grounded, the output current of the GB tied NMOSFET photodetector comes from the amplification of the $J_{1}$ photocurrent. When DNW is positively biased, the output current equals the sum of the amplification of the $J_{1}$ photocurrent and the amplification of the $J_{2}$ photocurrent. So the responsivity of the GB tied NMOSFET photodetector can be enhanced by the DNW structure.

\section{Results and Discussion}

Figure 2 shows the output current $\left(I_{d}\right)$ characteristics of the detector with $V_{\mathrm{ds}}$ under the same illumination of $2.0 \mu \mathrm{W} / \mathrm{cm}^{2}$. Four electrical measure conditions are included: (1) GB not tied with $V_{\mathrm{DNW}}=0 \mathrm{~V}$; (2) GB tied with $V_{\mathrm{DNW}}=0 \mathrm{~V}$; (3) GB tied with $V_{\mathrm{DNW}}=0.1 \mathrm{~V}$; and (4) GB tied with $V_{\mathrm{DNW}}=$ $0.5 \mathrm{~V}$. It can be seen that the output drain current of GB tied NMOSFET with $V_{\mathrm{DNW}}=0 \mathrm{~V}$ is two orders of magnitude higher than that of GB not tied NMOSFET. This result is in agreement with previous result [7] that the output current increase comes from the amplification of photocurrent of $J_{1}$ diode due to the gate-bulk tied structure. Compared to that of $V_{\mathrm{DNW}}=0 \mathrm{~V}$, the output current of the GB tied NMOSFET photodetector can be further increased one order

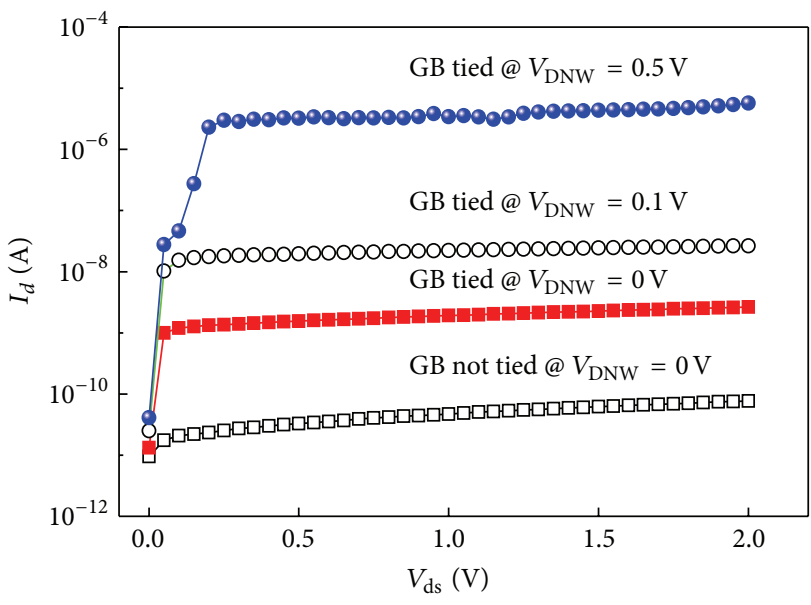

FIGURE 2: Output drain current characteristics of the photodetector under the same illumination of $2.0 \mu \mathrm{W} / \mathrm{cm}^{2}$. Four conditions are included: (1) GB not tied with $V_{\mathrm{DNW}}=0 \mathrm{~V}$; (2) GB tied with $V_{\mathrm{DNW}}=0 \mathrm{~V}$; (3) GB tied with $V_{\mathrm{DNW}}=0.1 \mathrm{~V}$; and (4) GB tied with $V_{\text {DNW }}=0.5 \mathrm{~V}$.

of magnitude when $V_{\mathrm{DNW}}=0.1 \mathrm{~V}$ and nearly four orders of magnitude when $V_{\mathrm{DNW}}=0.5 \mathrm{~V}$, informing that $V_{\mathrm{DNW}} \mathrm{can}$ greatly increase the output current.

We also characterize the relationship between the output current of the GB tied NMOSFET photodetector and the light intensity at $V_{\mathrm{ds}}=0.5 \mathrm{~V}$ as shown in Figure 3. The output current is increased in the whole range of light intensity when DNW is positively biased. The inset picture of Figure 3 shows $V_{\text {DNW }}$ dependence of the output dark current and photocurrent under light condition of $2.0 \mu \mathrm{W} / \mathrm{cm}^{2}$. It is found that the output photocurrent increases exponentially with $V_{\mathrm{DNW}}$ before $0.5 \mathrm{~V}$. Then, it remains essentially the same 


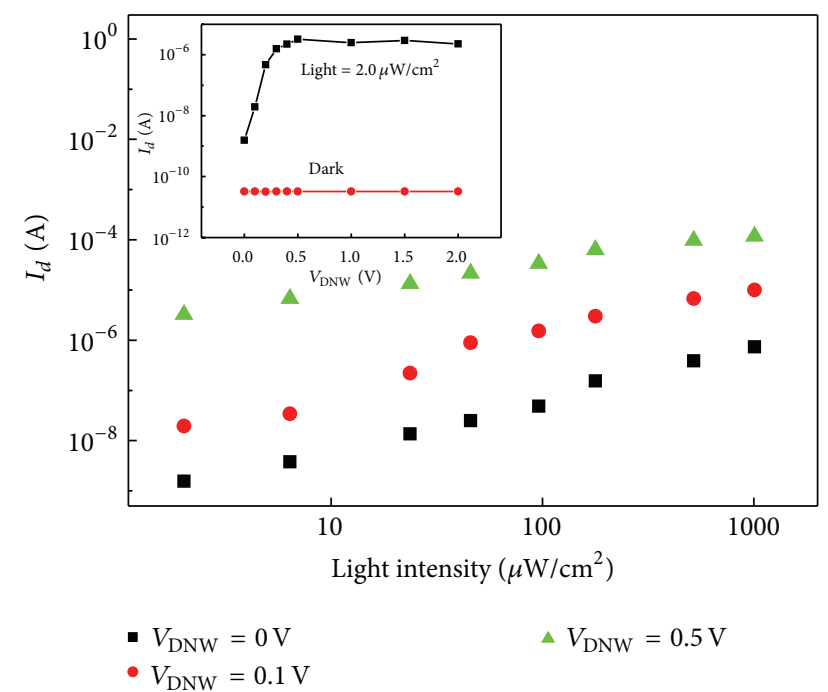

FIGURE 3: The relationship between output drain current and light intensity under different DNW voltages of the GB tied NMOSFET photodetector. The inset picture shows the $V_{\mathrm{DNW}}$ dependence of the output dark current and photocurrent under light condition of $2.0 \mu \mathrm{W} / \mathrm{cm}^{2}$. The output current is measured at $V_{\mathrm{ds}}=0.5 \mathrm{~V}$.

TABLE 1: Performance comparison of published detector [7] and our detector $\left(V_{\mathrm{ds}}=0.5 \mathrm{~V}\right.$ and light intensity $\left.=1 \mathrm{~mW} / \mathrm{cm}^{2}\right)$.

\begin{tabular}{cccc}
\hline Published & $\begin{array}{c}\text { Calculated } \\
\text { detector } \\
{[7]}\end{array}$ & $\begin{array}{c}\text { result } \\
\text { according to [7] }\end{array}$ & $\begin{array}{c}\text { Our detector } \\
\left(V_{\mathrm{DNW}}=0.5 \mathrm{~V}\right)\end{array}$ \\
\hline \multirow{3}{*}{ Photocurrent } & $0.1 \mu \mathrm{A}$ & $1.3 \mu \mathrm{A}$ & $110 \mu \mathrm{A}$ \\
& $(5 \mu \mathrm{m} \times 2 \mu \mathrm{m})$ & $(10 \mu \mathrm{m} \times$ & $(10 \mu \mathrm{m} \times$ \\
$0.55 \mu \mathrm{m})$ & $0.55 \mu \mathrm{m})$ \\
Dark current & $2.9 \mathrm{pA}$ & $38 \mathrm{pA}$ & $32 \mathrm{pA}$ \\
& $(5 \mu \mathrm{m} \times 2 \mu \mathrm{m})$ & $(10 \mu \mathrm{m} \times$ & $(10 \mu \mathrm{m} \times$ \\
& & $0.55 \mu \mathrm{m})$ & $0.55 \mu \mathrm{m})$ \\
\hline
\end{tabular}

when DNW voltage is beyond $0.5 \mathrm{~V}$. On the other hand, the dark current almost exhibits no change with the increased DNW voltage.

Table 1 lists the performance comparison of published detector [7] and our detector. We can see that the output photocurrent of our detector at $V_{\mathrm{DNW}}=0.5 \mathrm{~V}$ is about 2 orders of magnitude higher than the previous work under the same light intensity and the dark current almost does not change. It is therefore concluded that our photodetectors can be greatly improved by the DNW structure and have a larger responsivity than the previous work, while the dark current almost does not change. As analyzed above, the additional increase of output photocurrent of the photodetector when DNW is positively biased comes from the amplification of the photocurrent of $J_{2}$ diode.

To further analyze the characteristics of the responsivity enhanced photodetector, the photocurrents of $J_{1}$ and $J_{2}$ diodes before amplification are measured. Figure 4 shows the light intensity dependence of measured photocurrents of $J_{1}$ and $J_{2}$ at $V_{\mathrm{DNW}}=0.1 \mathrm{~V}$. The optically generated current of $J_{1}$ diode before amplification is achieved by operating

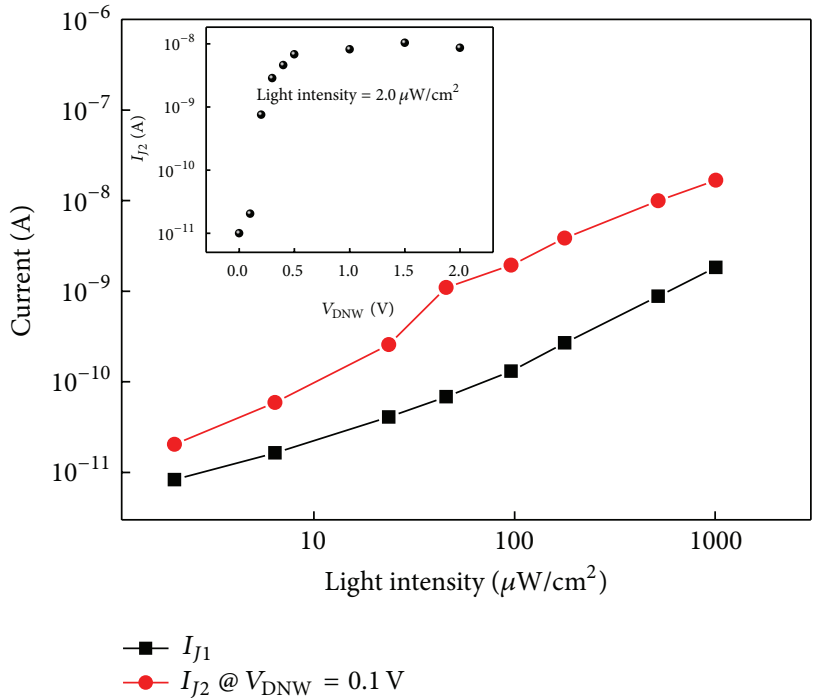

FIGURE 4: The light intensity dependence of the photocurrent of $J_{1}$ and $J_{2}$ diodes at $V_{\mathrm{DNW}}=0.1 \mathrm{~V}$. The inset picture shows the relationship between $I_{J 2}$ and $V_{\text {DNW }}$ under light condition of $2.0 \mu \mathrm{W} / \mathrm{cm}^{2}$.

the NMOSFET in the diode mode. Note that the measured photocurrent $I_{J 1}$ has to be halved to get the $J_{1}$ diode current before amplification [7]. The photocurrent $I_{J 2}$ of $J_{2}$ diode is measured at the DNW terminal with $V_{\mathrm{ds}}=0.5 \mathrm{~V}$ and $V_{\mathrm{DNW}}=0.1 \mathrm{~V}$ during photodetection. It can be seen that the photocurrent of $J_{2}$ diode is nearly one order higher than that of $J_{1}$ in the whole range of light intensity, which indicates that most of the holes accumulated in the bulk come from $J_{2}$. It is because the area of $J_{2}$ is very large and can absorb photons more efficiently than $J_{1}$. Therefore, the $J_{2}$ diode can greatly increase the gate potential of the NMOSFET and contributes much more to the output photocurrent of the photodetector than $J_{1}$ as the measured results shown in Figure 2.

The inset picture of Figure 4 shows the relationship between $I_{J 2}$ and $V_{\mathrm{DNW}}$ under the light condition of $2.0 \mu \mathrm{W} / \mathrm{cm}^{2}$. It can be seen that $I_{J 2}$ increases exponentially with $V_{\mathrm{DNW}}$ and then it is saturated when $V_{\mathrm{DNW}}$ exceeds $0.5 \mathrm{~V}$, which can explain the relationship between the output drain photocurrent and $V_{\mathrm{DNW}}$ as shown in the inset picture of Figure 3.

As discussed above, the output current of the GB tied NMOSFET photodetector comes from the amplification of $I_{I 1}$ when DNW is grounded, and it equals the sum of the amplification of $I_{J 1}$ and $I_{J 2}$ when DNW is positively biased. So we can calculate the gain factors of the two photodiodes, respectively.

The gain factor of $I_{J 1}$ is calculated using the following equation:

$$
\operatorname{Gain}_{J 1}=\frac{I_{d}\left(V_{\mathrm{DNW}_{0}}\right)}{0.5 I_{J 1}}
$$

where Gain ${ }_{J 1}$ is the gain factor of the $J_{1}$ diode photocurrent and $I_{d}\left(V_{\mathrm{DNW} 0}\right)$ is the output current measured with $V_{\mathrm{DNW}}=$ $0 \mathrm{~V}$. 
The gain factor of $I_{J 2}$ is calculated using the following equation:

$$
\operatorname{Gain}_{J 2}=\frac{I_{d}\left(V_{\mathrm{DNW}}\right)-I_{d}\left(V_{\mathrm{DNW} 0}\right)}{I_{J 2}}
$$

where Gain ${ }_{I 2}$ is the gain factor of the $J_{2}$ diode photocurrent and $I_{d}\left(V_{\mathrm{DNW}}\right)$ is the output current measured when DNW is positively biased with $V_{\mathrm{DNW}}$.

Figure 5 shows the calculated gain factor of the photocurrent of $J_{1}$ and $J_{2}$ diodes under different light intensity with $V_{\mathrm{DNW}}=0.1 \mathrm{~V}$. We can see that the gain factor of $I_{J 2}$ is larger than that of $I_{J 1}$ under low light condition. So our proposed new structure can efficiently enhance the responsivity of the photodetector under low light condition. It can also be found that the gain factor of $I_{J 1}$ increases at first and then decreases with illumination, while the gain factor of $I_{I 2}$ is very large under low light condition and then decreases with illumination. It is because of that that the $J_{1}$ photocurrent is very small under low light condition and little holes are accumulated in the bulk. So the NMOSFET stays in depletion region and the channel surface potential increases quickly with the increased gate potential (induced by the accumulated holes). Therefore, the gain factor of $I_{J 1}$ increases with the light intensity at first. But when the light intensity becomes large, the output drain current increases and the NMOSFET enters into strong inversion gradually. The surface potential of the NMOSFET changes very little when the gate potential continued increase [8]. So the gain factor of $I_{J 1}$ decreases when the light intensity continues to increase. On the other hand, for $\mathrm{J}_{2}$ diode, because of the large gain factor and the large photon-absorbing efficiency of $J_{2}$ when $V_{\mathrm{DNW}}=0.1 \mathrm{~V}$, the NMOSFET has entered into inversion region under low light condition. So the surface potential of the NMOSFET changes very little when the light intensity continues to increase and the gain factor of $J_{2}$ decreases with the light intensity as shown in Figure 5.

\section{Conclusion}

In this paper, one responsivity enhanced NMOSFET photodetector on a DNW is studied. Because of the DNW structure, the output photocurrent of the enhanced photodetector comes from two parts. One is from the amplification of the drain/bulk diode photocurrent and the other is from the amplification of the DNW/bulk diode photocurrent. Studies indicate that the photon-absorbing efficiency of the DNW/bulk diode is very high and most of the holes accumulated in the bulk come from the DNW/bulk diode. So the DNW/bulk diode contributes most of the output optical current and can greatly improve the responsivity. The experimental results show that the responsivity of our detector can be enhanced greatly by the DNW structure and the photocurrent is nearly 2 orders of magnitude higher than the previous work when DNW is biased with $0.5 \mathrm{~V}$ under the illumination of $1 \mathrm{~mW} / \mathrm{cm}^{2}$, while the dark current exhibits almost no increase. Meanwhile, the gain factor of the photocurrent of DNW/bulk diode is very large under low light condition, which can efficiently enhance the responsivity of

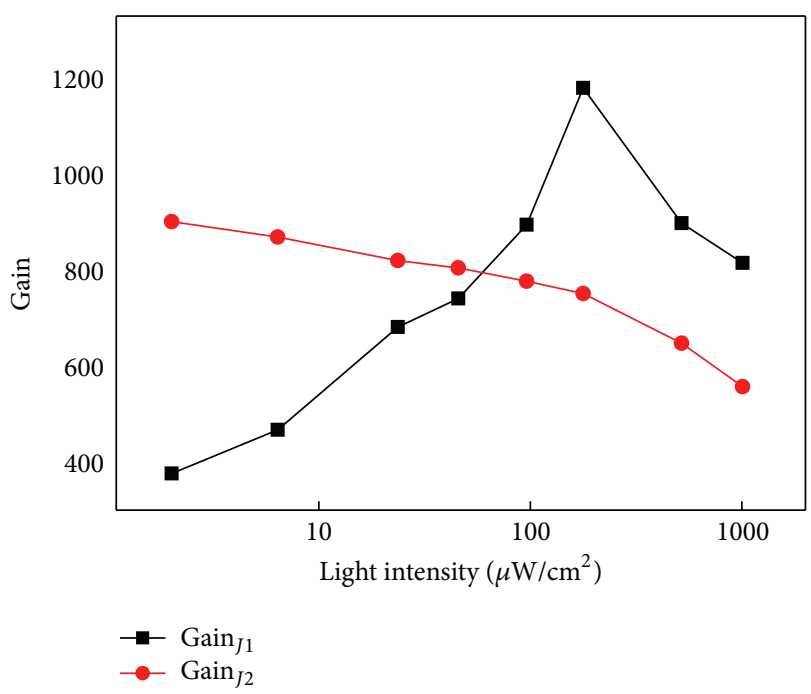

Figure 5: The gain factors of the photocurrent of $J_{1}$ and $J_{2}$ diodes under different light conditions with $V_{\mathrm{DNW}}=0.1 \mathrm{~V}$.

the detector under low light condition and makes it very suitable for low light detecting.

\section{Conflict of Interests}

The authors declare that there is no conflict of interests regarding the publication of this paper.

\section{Acknowledgment}

This work is partially supported by Graduate Student Training Innovative Project of Jiangsu Province CXZZ13_0052.

\section{References}

[1] Z. Ignjatovic, D. Maricic, and M. F. Bocko, "Low power, high dynamic range CMOS image sensor employing pixel-level oversampling $\Sigma \Delta$ Analog-to-digital conversion," IEEE Sensors Journal, vol. 12, no. 4, pp. 737-746, 2012.

[2] M. Bigas, E. Cabruja, J. Forest, and J. Salvi, "Review of CMOS image sensors," Microelectronics Journal, vol. 37, no. 5, pp. 433451, 2006.

[3] N. Faramarzpour, M. El-Desouki, M. J. Deen, Q. Fang, S. Shirani, and L. W. C. Liu, "CMOS imaging for biomedical applications," IEEE Potentials, vol. 27, no. 3, pp. 31-36, 2008.

[4] K. B. Mogensen, H. Klank, and J. P. Kutter, "Recent developments in detection for microfluidic systems," Electrophoresis, vol. 25, no. 21-22, pp. 3498-3512, 2004.

[5] O. Tigli, L. Bivona, P. Berg, and M. E. Zaghloul, "Fabrication and characterization of a surface-acoustic-wave biosensor in CMOS technology for cancer biomarker detection," IEEE Transactions on Biomedical Circuits and Systems, vol. 4, no. 1, pp. 62-73, 2010.

[6] H. Yamamoto, K. Taniguchi, and C. Hamaguchi, "Highsensitivity SOI MOS photodetector with self-amplification," Japanese Journal of Applied Physics, vol. 35, part 1, no. 2B, pp. 1382-1386, 1996.

[7] W. Zhang, M. Chan, and P. K. Ko, "Performance of the floating gate/body tied NMOSFET photodetector on SOI substrate," 
IEEE Transactions on Electron Devices, vol. 47, no. 7, pp. 1375$1384,2000$.

[8] S. M. Sze, Physics of Semiconductor Devices, John Wiley \& Sons, Hoboken, NJ, USA, 3rd edition, 2007. 

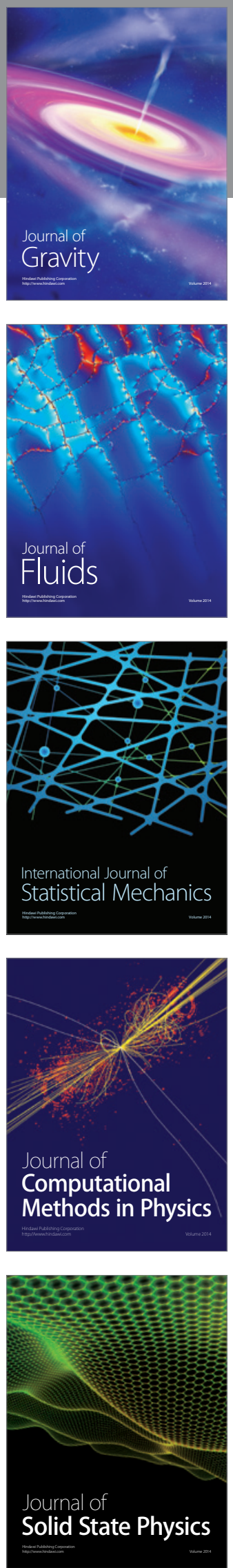

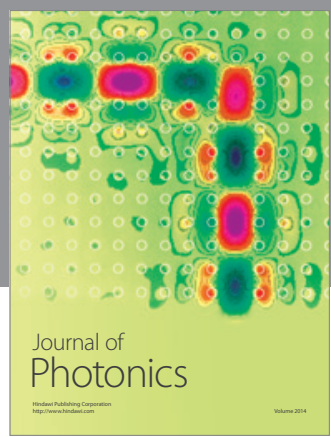

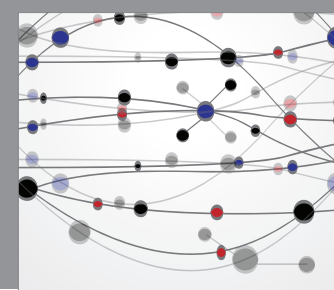

The Scientific World Journal

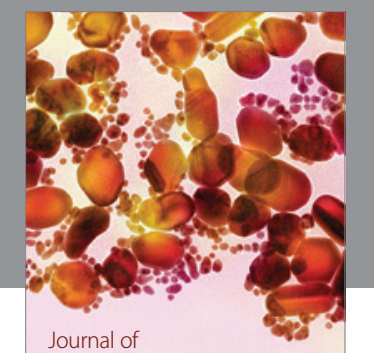

Soft Matter
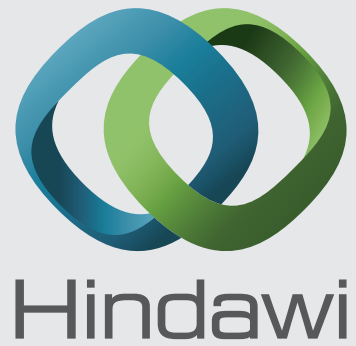

Submit your manuscripts at

http://www.hindawi.com
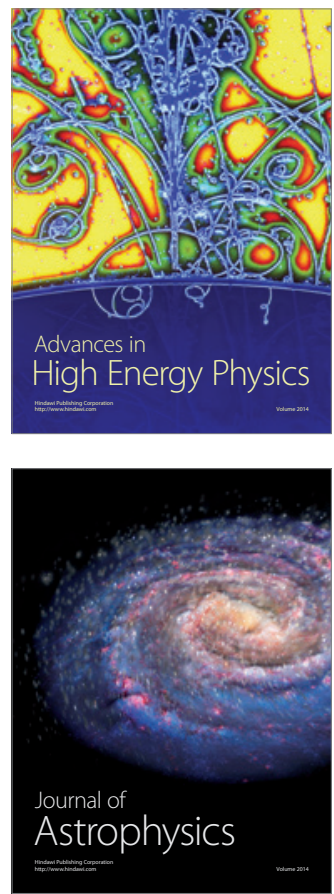
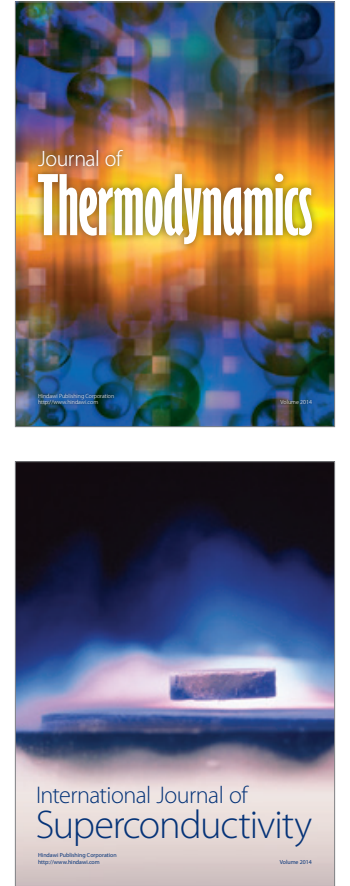
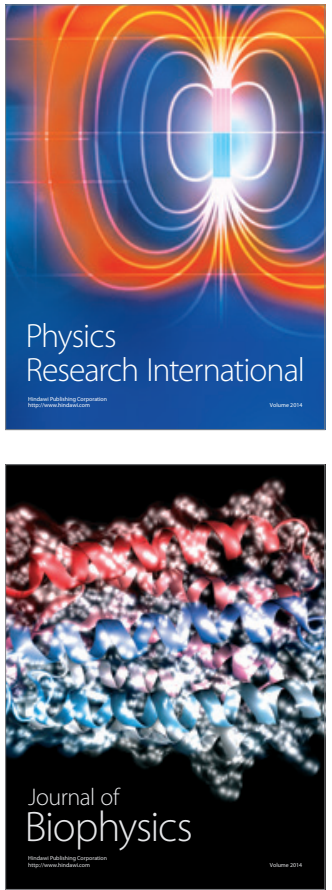
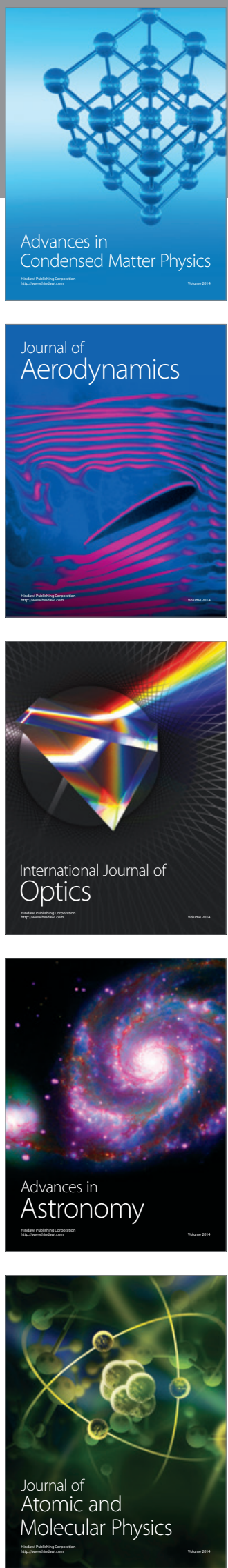